\title{
Teaching/Learning Astronomy at the Elementary School Level
}

\author{
By Nicoletta Lanciano \\ Dipartimento di Matematica, Università "La Sapienza", Rome, Italy
}

\section{What kind of astronomy can be taught to children between the ages of 6 and 11 ?}

There are those who argue that children have little familiarity with the sky and that the study of astronomy should be put off until they're older. We believe, on the other hand, that children have an intimate daily rapport with the sky, the sun and moon especially, based on genuine affection for these celestial bodies which is often expressed in their fantasies, reminiscent of ancient mythology and present-day primitive cultures. Their initial conceptions of celestial objects and phenomena bring to mind ancient philosophical conceptions and the kind of erroneous thinking induced by present-day culture and mass media, and make us aware of how difficult it is to develop personal perceptions and of the powerful emotions that prevent or inhibit us from building new ones. The kind of astronomy we present to young children, with which we have been experimenting for years, is not the kind usually taught in schools and cannot be broken down into various different topics. We have children observe nature, do real life drawings of it, concentrate on it and listen to mythological stories so as to sensitize them to the rhythm of sounds, song, motion, numerical calculation and geometric representation.

This kind of astronomy only deals with what can be seen and recorded with the naked eye: the Earth, Sun, Moon, Venus, Mars, Jupiter and Saturn, the constellations and the sky, a theater of celestial bodies in motion.

\section{ASTRONOMY FOR CHILDREN}

- IS NOT A SUBJECT MATTER AS SUCH

- IS NOT BROKEN DOWN INTO TOPICS

- ONLY CONSIDERS CELESTIAL BODIES VISIBLE IN THE SKY TO THE NAKED EYE

\section{RECITING MYTHS ASTRONOMY GEOMETRY}

WE CAN TELL FROM THE NAMES OF CONSTELLATIONS WHETHER THEY ARE IN THE NORTHERN OR SOUTHERN HEMISPHERE.

\section{Research Methods}

We will present:

a) methods for researching children's initial conceptions;

b) activities for interacting with these conceptions, and a comparison with other teaching methods;

c) how these conceptions evolved in groups of children between 6 and 11 under our observation. 


\section{TEACHING ACTIVITIES DESIGNED TO IDENTIFY INITIAL CONCEPTIONS, but which also \\ ENCOURAGE THEIR EVOLUTION \\ and \\ TEACHING ACTIVITIES DESIGNED TO INTERACT \\ WITH INITIAL CONCEPTIONS AND CONCEPTION BLOCKS, \\ but which also \\ IDENTIFY NEW INITIAL CONCEPTIONS}

The following are different activities designed to elicit different kinds of perceptions:

- working with clay involves the hands

- doing theater involves the whole body

- dialogue makes use of words which are spoken and listened to (oral)

- individual and group writing makes use of words which are written down and read.

- drawing makes use of sight and hands.

Information is thus gathered in the form of:

- objects produced

- theatrical actions or gestures on the part of individual children

- written texts produced by individuals or the group

- individual drawings

While thinking in terms of products, special attention is paid to the processes which groups or individual children go through in their work.

\subsection{Children's Initial Conceptions of the Sun}

The following are some of the conceptions children have of the Sun, expressed in conversation, mime and drawings.

Many children say that: "The Sun has rays."

Small children think that "at night the rays go back in": they envisage transformations in the natural world with great nonchalance and sense of dynamics. Some of them think that "at night the Sun turns into the moon". Leaving aside what is incorrect, the important thing is to maintain this sense of motion, of dynamics, of transformation: the Sun, being a star, is a body in continual transformation. The problem in terms of teaching is to get this initial concept to evolve in the desired direction.

In terms of these rays, there is a new obstacle:

- we see diverging rays leaving the Sun's disk

- we have the children consider this for a moment and then say that "they are parallel to each other when they reach the Earth"

There is no one correct response. It is not that one of them is right and the other wrong, so on the one hand it is a question of combining reflection on macrospace (the Earth and Sun in which we see diverging rays coming out of the Sun's disk), and mesospace (the Earth's ground where these rays hit and can be said to be parallel to each other), and on the other, we need to introduce the fact that light moves in straight lines and is propagated in all directions, implying that we can intercept a ray, thus introducing the question of diffused light. In this context megaspace is the great celestial space which is inaccessible to us and which we can see only in part, and mesospace is the space we move in, totally accessible to us both physically and visually. There is also what we refer to as microspace, which is close, totally accessible to our eye, and manipulable, and macrospace, which is immense, partially accessible to the eye and inaccessible to us in terms of movement.

$$
\text { MACROSPACE OF THE EARTH AND THE SUN }
$$

IN WHICH WE SEE THE SUN'S DIVERGING RAYS COME OUT OF ITS DISK MESOSPACE OF THE EARTH'S SURFACE

WHERE THE RAYS HIT (WHICH CAN BE CONSIDERED) ALL PARALLEL TO EACH OTHER 
CRUCIAL IMPORTANCE OF STATING IMPLICIT FRAMES OF REFERENCE

PROBLEMS OF LIGHT: children think

- THE RAYS GO CROOKED or THE RAYS GO STRAIGHT

Light:

- GOES IN A STRAIGHT LINE

- RADIATES IN ALL DIRECTIONS

- IT IS POSSIBLE TO INTERCEPT A RAY

- EXPERIENCE WITH DIFFUSED LIGHT

\section{EXPANDING OUR FIELD OF VALIDITY THE SUN IS A DISK \\ THE SUN IS A SPHERE THAT EMITS LIGHT \\ I SEE A DISK - I IMAGINE A SPHERE THE SAME AS FOR THE MOON}

\subsubsection{Example of a conception that evolves with age (regardless of formal education)}

Elementary school children from 6 to 7 often maintain that "there are a lot of suns", of different colors, sizes, positions, that can be seen in different parts of the world, etc. They often draw more than one sun in the sky and more than one moon. At the symbolic, unconscious level, and not as a simple expression of knowledge, this could mean that the Sun is important, that it symbolizes festivity.

\section{MANY SUNS JUST AS THERE ARE MANY EARTHS (THE PLANET EARTH/ THE EARTH WHERE WE LIVE) JUST AS THERE ARE MANY MOONS}

to

ONLY ONE SUN

\subsubsection{Activities designed to interact with conceptions, encourage their evolution and avoid certain teaching obstacles}

Our shadows: shadows are not just on the ground but occupy space. To make this clear, we give examples to show that we are all aware of this fact in our daily lives and, for example, park our cars out of the Sun and sit under beach umbrellas. The difficulty, in this case, is to get people to consider everyday experiences when they are thinking scientifically, in a "scholastic"situation in which, due to erroneous teaching, shadows are usually considered in terms of projections on horizontal planes (the ground) or vertical planes (a wall). So what we do is to fill our shadows with branches or with other people's bodies, we divide them into sections with white sheets of cloth. This way we can see that shadows are in space, that they occupy three-dimensional space, and that they can have longer or shorter sections which are deformed if we tilt the cloth.

Throughout this work, in keeping with what will follow concerning deep and emotional elements, we work with the shadows of our own bodies and not the usual shadow of a telephone pole. We feel that as a first approach to the Sun and shadows, this is much more advantageous, not only because it allows students to study the volume of a shadow, but also because it is attached to them. We always have our shadows with us, even when we're not in school, on the beach for example, so we can continue to make discoveries, on our own, comparing them with others, experimenting, etc.

A monument of bamboo canes to trace the Sun's path: during an entire day of hands-on work, observation and reflection, we construct a three-dimensional track in space of the Sun's path in the sky. At the end of the day we have an arc, which may be of varying height, covered in colored disks, showing the path the Sun took that day. The fact that this group activity 
takes a long time gives everyone the possibility of understanding the sense of what is being done and of formulating questions more and more precisely and to the point. From elements observed in the "here and now", we can form mental images of what can be seen elsewhere or at other times of the year. This gives us a foundation of direct observation and an imaginative and precise record of it with which to reason about things we can see only in our mind's eye.

\author{
THE SUN'S PATH WITH BAMBOO CANES and not GNOMON \\ - TO AVOID ERRORS ABOUT THE DIRECTION OF THE SUN AND THE \\ DIRECTION OF SHADOW \\ - TO AVOID PROBLEMS OF INDIRECT VISION: SUN FROM EAST TO WEST \\ SHADOW FROM WEST TO EAST \\ - TO MAKE SURE THAT THE WORK IS CARRIED OUT OVER A LONG \\ PERIOD OF TIME, WITHOUT ANTICIPATING, WITHOUT SPEAKING \\ INSTEAD OF OBSERVING, WITHOUT EXPLAINING INSTEAD \\ OF EXPERIENCING
}

\title{
2.1.3. Evolution of conceptions as the expansion of our field of validity
}

By evolution of conceptions in students we consider the fact that their conceptions go from a simple level to a more complex one in which they are capable of considering multiple aspects. This does not mean that the new conception will be more "correct"in the scientific sense; on the contrary, greater complexity often leads to confusion, but the conceptual field of validity will be wider.

\section{Training courses for teachers}

We address the question of initial teacher training and updating courses for teachers at the elementary school level in Italy by presenting several examples of activities carried out in residential courses or in the university, and the problems encountered. Elementary school teachers in Italy are not required to have a degree, and in any case almost none of them has one in a scientific subject. The only scientific background they have is what they receive in high school.

In these courses we work on:

- the geometry of the sky: the great celestial and terrestrial circles: the equator, the horizon, meridians, ecliptics, etc., the height of their angles, etc.

- music, to find connections between what we observe and study about the sky and music, sound, rhythm, song and how musical instruments are made

- motion that helps us have a perception of ourselves, our bodies and the space around us

- myths and experiences with other cultures (myths about the constellations and planets)

- designing and making instruments by hand

0 - for observing and recording celestial phenomena

- - in order to have a clear, precise image stamped on our memories necessary because usual written texts with two-dimensional drawings and spoken lessons on complex phenomena have proven to be difficult to follow and to have little to do with our intuitions, emotions and personal effort.

In proposing this kind of teaching we remind people that the sky is everywhere and available to everyone, even poorly equipped schools in underdeveloped areas. This means that everyone can study astronomy simply by using the sky as a private research laboratory. And this kind of contact with the sky encourages children to be concerned about nature and the environment instead of wanting to manipulate and exploit it and science. It is our belief that the study of astronomy can contribute to re-establishing relationships between individuals, the environment and the planet.

In terms of method, it is important to keep in mind:

- the richness of mythology; 
- the value of direct observation in the open,

- the value of long periods of systematic observation,

- the importance of addressing a subject from a cross-curricular point of view and not limiting it to traditional science;

- the attention paid to participants' initial concepts,

- the advantages of working together with our minds and hands to create instruments which can record three-dimensional celestial phenomena observed.

In both types of courses, local and outside of schools, we work on primary and fundamental elements.

\subsection{Primary and fundamental elements}

We work on primary cognitive elements which constitute the foundation of knowledge and that go back to the stage of learning in which understanding is thinking and doing, touching, moving, being thrilled.

Primary elements of the sky are, for example

The Local Horizon

The real local horizon (Lanciano et al 1996) can be seen everywhere out in the open: it is the dividing line between the sky and the Earth. If we stand in one spot and turn $360^{\circ}$ we can trace the entire horizontal line with our gaze. It is generally not a circle and is usually not all level; in fact it is usually very uneven, and if we are in a city there will be buildings, light poles, antennas, maybe some hills, as well as trees. This is our real horizon which determines what time we can see the Sun in different periods of the year and what time it will set, i.e. disappear over the horizon. The astronomical, theoretical horizon is not useful when we are trying to decide what to observe in the sky and when, so we have to learn to consider our local horizon. This is why we observe it, draw it and use it to create our observatory.

\section{The Globe}

The same globe is used in all countries, but the inclination of its axis is not clear and significant to everybody. This is why we take it off its support when introducing it and set it in the position of a "parallel map"(Lanciano et al 1996), i.e. tilting its axis according to the latitude of a place, that is, directed toward the North Star. If Italy is at the top of the globe, with its tangential plane parallel to the table on which it sits, the Sun will do exactly what it does on Earth. We can see which pole is in darkness and which is illuminated, which countries are in daytime and where the Sun is at its zenith. This allows us to feel "central in relation to what we are observing", to become aware of our own particular vantage point and to connect the Earth we walk on with the globe we look at from outside.

This instrument stimulates us to think about what we feel to be our position on the Earth's sphere. We discover how much the fact that we think of North as high and South as low leads to unconscious conceptual and "geometrical" errors. We discover something new about the "sphericity"- physical, geometrical, astronomical and geographical - of our planet.

Meridians

A local meridian is every North-South line, and it is possible to find one at every point of the Earth's surface; meridians are not only those marked on the globe or map - theoretically it is possible to connect the two poles at any point on the globe. The problem involved in this kind of change in thinking is once again the problem of moving from the discrete to the continuum: from a discrete grid to great circles - to continuum circles which theoretically cover the entire surface of the sphere.

We work with fundamental elements because they involve the whole person. Some examples are painting, reciting and listening to myths, sounds, songs, becoming familiar with the night and celestial bodies (Lanciano 1993, Montinaro). In all these arts, these expressive languages, our hands, bodies, thoughts, emotions and hearts are directly involved. It is our conviction that it is impossible to follow a path of knowledge if we are resisting it in any way, if we are not ready to meet the unknown, something that could change our scheme of things, our basis of security. Learning always involves abandonment of the known, what we feel is ours which may be incorrect or incomplete but which reassures us. Imagine how difficult and frightening 


\section{N. Lanciano: Teaching/Learning Astronomy at the Elementary School Level}

it must be for a small child to give up the comforting idea of a nice flat world for a spherical one in motion, suspended in the void like the moon and planets in the sky. Every discovery and conquest our minds achieve is accompanied by emotion, and the quality of the relationship we have with those who accompany and guide us and the objects we are studying determine whether our knowledge will be stable or uncertain, whether it will constitute a basis for new questioning and discoveries or will be a series of disconnected notions, easily forgotten. This is what psychology is telling us about the learning process, but it seems to be immediately forgotten or ignored when it comes to teaching individual subjects, when knowledge is broken up into separate subjects, when our main purpose in education becomes evaluating instead of teaching and offering opportunities for growth and discovery.

One of the most important factors in our work is that those who guide students be not only competent, but passionate about what they are offering to others and about education itself.

\section{REFERENCES}

Lanciano, N., 1993, Il suono e le stelle, Cooperazione Educativa n. 6, La Nuova Italia ed, Florence.

Lanciano, N. ET al Dentro il cielo lecture notes, Quaderno n. 7 of the L.D.S., 1996.

Montinaro, R. Le vie dei suoni, Il Crogiolo. 